\title{
New Method for Dynamical Fermions and Chiral-Symmetry Breaking
}

\author{
V. Azcoiti ${ }^{a}$, G. Di Carlo ${ }^{\mathrm{b}}$, A.F. Grillo ${ }^{\mathrm{b}}$ V. Laliena ${ }^{\mathrm{a}}$ and X.Q. Luo ${ }^{\mathrm{a}} *$ \\ a Departamento de Física Teórica, Facultad de Ciencias, Universidad de Zaragoza, \\ 50009 Zaragoza, Spain

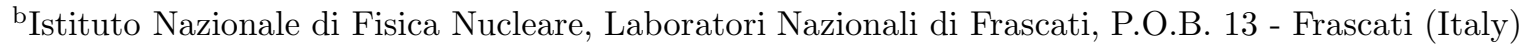

The reasons for the feasibility of the Microcanonical Fermionic Average $(M F A)$ approach to lattice gauge theory with dynamical fermions are discussed. We then present a new exact algorithm, which is free from systematic errors and convergent even in the chiral limit.

\section{INTRODUCTION}

The dynamics of a gauge-fermion system on a $d$-dimensional lattice is described by its action

$$
S=-\beta S_{p l}(U)-N_{f}^{L} \ln \operatorname{det} \Delta(m, U),
$$

where the fermionic degrees of freedom have been integrated out, and $N_{f}^{L}$ is number of flavors taking into account the species doubling. $S_{p l}$ is the plaquette energy $\left[S_{p l}=\sum_{p} \operatorname{Re}\left(U_{p}\right) / N_{c}\right.$, for a compact theory, and $S_{p l}=-\sum_{p} \theta_{p}^{2} / 2$, for noncompact $Q E D]$.

The fermionic determinant $\operatorname{det} \Delta(m, U)$ accounts for the effects of dynamical fermions; its numerical evaluation requires in general a huge $C P U$ time due to nonlocality and dependence on different bare parameters.

Therefore, one of the most challenging tasks in lattice simulations is the inclusion of dynamical fermions. Furthermore, most of the conventional algorithms can not be applied to the chiral limit $(m=0)$ because of the problem of convergence.

In this paper, we discuss two efficient approaches to simulating dynamical fermions which are convergent even in the chiral limit: the Microcanonical Fermionic Average (MFA) method and a new exact algorithm.

\footnotetext{
*Presented by X.Q. Luo. This work has been partly supported through a INFN(Italy)-CICYT(Spain) collaboration. X.Q. Luo acknowledges support from the Ministerio de Educacion y Ciencia.
}

\section{FEASIBILITY OF THE MFA METHOD}

The main point of the $M F A$ approach [1] to dynamical fermions is to compute the full effective action as a function of the pure gauge energy $E$ and bare parameters $\left(m, N_{f}\right.$, and $\left.\beta\right)$ :

$$
\begin{aligned}
& S_{e f f}\left(E, m, N_{f}, \beta\right)=-\ln N(E)-\frac{d(d-1)}{2} V \beta E \\
& +S_{\text {eff }}^{F}\left(E, m, N_{f}\right),
\end{aligned}
$$

where

$N(E)=\int[d U] \delta\left(S_{p l}(U)-\frac{d(d-1)}{2} V E\right)$

is the density of states at fixed $E$ and

$S_{e f f}^{F}\left(E, m, N_{f}\right)=-\ln \left\langle[\operatorname{det} \Delta(m, U)]^{N_{f}^{L}}\right\rangle_{E}$

is the effective fermionic action computed as a microcanonical average over the probability distribution

$\frac{\delta\left(S_{p l}(U)-\frac{d(d-1)}{2} V E\right)}{N(E)}$.

Then the partition function becomes a onedimensional integral

$Z=\int d E e^{-S_{e f f}\left(E, m, N_{f}, \beta\right)}$.

Once the full effective action as a function of $E$ and other bare parameters is known, the dynamics of the system can be investigated numerically 
or analytically. There are several advantages in this approach:

(a) $S_{\text {eff }}^{F}$ does not depend on $\beta$;

(b) The dependence on $N_{f}^{L}$ is trivial;

(c) $S_{\text {eff }}^{F}$ can be simultaneously computed for any $m$ (and particularly for $m=0$ ) if the Lanczos algorithm is employed to evaluate $\operatorname{det} \Delta$.

Now the most important question is how to compute $S_{\text {eff }}^{F}$ defined by (4). Since the configuration is sampled with the flat distribution (5), it may require high statistics to have the statistical errors in det $\Delta$ under control. A more reliable way is to perform a cumulant expansion, which turns out to be an expansion on $N_{f}^{L}$

$-S_{\text {eff }}^{F}\left(E, m, N_{f}\right)=N_{f}^{L}\langle\ln \operatorname{det} \Delta\rangle_{E}$

$+\left(N_{f}^{L}\right)^{2}\left\langle\left(\ln \operatorname{det} \Delta-\langle\ln \operatorname{det} \Delta\rangle_{E}\right)^{2}\right\rangle_{E}+\ldots$,

where the first term is some kind of mean field approximation, and the fluctuations correspond to the first corrections.

As is well known, the mean field approximation is a good one if the interaction is long ranged or the dimensionality is large. In [2], we have given explicit physical examples (the local Ising model, the nonlocal Ising model, the pure gauge, mixed compact-noncompact model etc.) to show how the locality and dimensionality play a role. In the nonlocal Ising model, only the first contribution to the cumulant expansion exists.

In the most interesting application of the $M F A$ method, the gauge-fermion system, because of the nonlocality of $\operatorname{det} \Delta$, it is expected that the cumulant expansion (7) converges rapidly, which has indeed been observed in all the simulations 33 10] of lattice gauge theory with dynamical fermions $\left(Q E D_{2}, Q E D_{3}, Q C D_{3}\right.$ and $\left.Q E D_{4}\right)$. For instance [2], in noncompact $Q E D_{4}$, the first cumulant is dominant, the second one is $2 \%$ of the first one, and the third one is compatible with zero. Using the $M F A$ method, the results for the Schwinger model [3] and for $Q E D_{4}[8,10]$ are in agreement with the exact calculable ones and consistent with those obtained with the Hybrid Monte Carlo algorithm $(H M C)$ respectively. The simulation of $Q C D_{4}$ is in progress.

\section{EXACT ALGORITHM FOR THE CHIRAL LIMIT}

As mentioned previously, the feasibility of the MFA method depends on the convergence of the cumulant expansion. In practice, only the first few cumulants can be reliably calculated and a truncation of the expansion has to be made. Since a good control of systematic errors is difficult, especially when $N_{f}$ is large, it seems interesting to us to develop some exact algorithm for obtaining quantitative results.

An efficient exact algorithm for dynamical fermions at $m \neq 0$ does exist, i.e. the $H M C$ algorithm. In order to study the chiral properties of lattice gauge theory without introduction of the external field $m \bar{\psi} \psi$ or mass extrapolations, it is important to construct an exact algorithm which is convergent even in the $m \rightarrow 0$ limit. To our knowledge, the Lanczos algorithm is the only efficient technique for evaluating $\operatorname{det} \Delta$ which is convergent in this limit. The problem with this algorithm is that, if the canonical simulation is carried out by using the probability distribution $e^{-\left(S_{g}-N_{f}^{L} \ln \operatorname{det} \Delta\right)}$ for each link update then the computer time is prohibitive; if many links are updated before testing for acceptance, then its rate is very low.

Here we present a new exact algorithm, based on the following decomposition of the full action

$S=S_{g}+S_{e f f}^{F}+\left(-N_{f}^{L} \ln \operatorname{det} \Delta-S_{e f f}^{F}\right)$,

where $S_{e f f}^{F}$ is a local effective action chosen to improve the acceptance rate of the canonical simulation, and the partition function can be rewritten as

$Z=\int[d U] e^{-\left(S_{g}+S_{e f f}^{F}\right)} e^{-\left(-N_{f}^{L} \ln \operatorname{det} \Delta-S_{e f f}^{F}\right)}$.

This exact algorithm is implemented as follows.

(a) Generate a new configuration $U^{\prime}$ with the probability distribution $e^{-\left(S_{g}+S_{e f f}^{F}\right)}$, which can be done efficiently and is equivalent to a effective pure gauge theory if we choose $S_{e f f}^{F}$ as that in Sect. 2.

(b) Accept $U^{\prime}$ according to the probability

$P_{A}=\min \left\{1, \frac{e^{N_{f}^{L} \ln \operatorname{det} \Delta\left(m, U^{\prime}\right)+S_{e f f}^{F}\left(m, U^{\prime}\right)}}{e^{N_{f}^{L} \ln \operatorname{det} \Delta(m, U)+S_{e f f}^{F}(m, U)}}\right\}$, 
Table 1

Acceptance rate of the exact algorithm

\begin{tabular}{lcrrrr}
\hline & \multicolumn{2}{c}{$S_{\text {eff }}^{F}=0$} & & \multicolumn{2}{c}{$S_{\text {eff }}^{F}$ obtained by $M F A$} \\
\cline { 2 - 3 } \cline { 5 - 6 } & $m=0$ & $m=0.02$ & & $m=0$ & $m=0.02$ \\
\hline$N_{f}=2, \beta=0.3$ & $26 \%$ & $36 \%$ & & $40 \%$ & $60 \%$ \\
\hline$N_{f}=4, \beta=0.25$ & $14 \%$ & $15 \%$ & & $26 \%$ & $30 \%$ \\
\hline
\end{tabular}

which is just the Metropolis test.

It can be shown that this approach is free from systematic errors and that detailed balance is satisfied. Furthermore this algorithm is convergent even in the chiral limit, since $\operatorname{det} \Delta$ is evaluated by the Lanczos method.

According to (10), the acceptance rate is higher if $S_{e f f}^{F}$ is a good local approximation to the fermionic action. Here we choose $S_{e f f}^{F}$ as that in the MFA method discussed in Sect. 2.

We have tested these ideas in noncompact $Q E D_{3}$ using this new exact algorithm. To avoid huge changes in $\ln \operatorname{det} \Delta$, we change only part of the links in each iteration (a). The results for the $10^{3}$ lattice (only 1000 links are systematically changed when $U^{\prime}$ is generated) are the following: (i.) For quenched $\left(N_{f}=0\right)$ and $N_{f}=2$ theories, the data for the chiral condensate $\langle\bar{\psi} \psi\rangle$ at nonzero mass agree with those 11] obtained by the hybrid algorithm.

(ii.) The results for $N_{f}=4$ are slightly different from those in 111. This is not surprising because systematic errors increase as $O\left(N_{f}^{2}\right)$ in the hybrid algorithm.

(iii.) In the canonical simulations, we find that the choice of $S_{\text {eff }}^{F}$ plays an important role in the improvement of the acceptance rate. Table 11 shows the results for the acceptance rate for different bare parameters. The data for direct canonical simulations $\left(S_{\text {eff }}^{F}=0\right)$ are also included in this Table. As one sees, the use of the effective fermionic action obtained by the $M F A$ method gives much better results for this quantity (it increases by about $40 \%$ ).

\section{CONCLUSIONS}

To summarize, we have argued that one of the most important reasons for the feasibility of the $M F A$ approach is the nonlocality of $\operatorname{det} \Delta$. Based on the $M F A$ method, a new exact algorithm, which is convergent even at $m=0$ is presented and possible ways of increasing the acceptance rate are discussed. An interesting application of this exact algorithm, the investigation of the vacuum chiral properties directly at $m=0$ by calculating the probability distribution function of the chiral condensate, can be found in [12].

\section{REFERENCES}

1. V. Azcoiti, G. Di Carlo and A.F. Grillo, Phys. Rev. Lett. 65 (1990) 2239.

2. V. Azcoiti, V. Laliena, X.Q. Luo, C.E. Piedrafita, G. Di Carlo, A. Galante, A.F. Grillo, L.A. Fernandez and A. Vladikas, Phys. Rev. D48 (1993) 402.

3. V. Azcoiti, G. Di Carlo, A. Galante, A.F. Grillo and V. Laliena, these proceedings.

4. V. Azcoiti and X.Q. Luo, Nucl. Phys. B(Proc. Suppl.)30 (1993) 741.

5. V. Azcoiti and X.Q. Luo, DFTUZ.92/25, to appear in Mod. Phys. Lett. A.

6. V. Azcoiti, X.Q. Luo, C.E. Piedrafita, G. Di Carlo, A.F. Grillo and A. Galante, Phys. Lett. B313 (1993) 180.

7. V. Azcoiti and X.Q. Luo, Phys. Lett. B313 (1993) 191.

8. V. Azcoiti, A. Cruz, G. Di Carlo, A.F. Grillo and A. Vladikas, Phys. Rev. D43 (1991) 3487.

9. V. Azcoiti, G. Di Carlo and A.F. Grillo, Mod. Phys. Lett. A7 (1992) 3561.

10. V. Azcoiti, G. Di Carlo and A.F. Grillo, Phys. Lett. B305 (1993) 275.

11. E. Dagotto, A. Kocic and J. Kogut, Nucl. Phys. B334 (1990) 279.

12. V. Azcoiti, G. Di Carlo, A.F. Grillo, V. Laliena and X.Q. Luo, these proceedings. 